\title{
Is Travel Demand Actually Deep? An Application in Event Areas Using Semantic Information
}

Markou, loulia; Rodrigues, Filipe; Pereira, Francisco Camara

Published in:

I E E E Transactions on Intelligent Transportation Systems

Link to article, DOI:

10.1109/TITS.2019.2897341

Publication date:

2020

Document Version

Peer reviewed version

Link back to DTU Orbit

Citation (APA):

Markou, I., Rodrigues, F., \& Pereira, F. C. (2020). Is Travel Demand Actually Deep? An Application in Event Areas Using Semantic Information. I E E E Transactions on Intelligent Transportation Systems, 21(2), 641-652. [19320350]. https://doi.org/10.1109/TITS.2019.2897341

\section{General rights}

Copyright and moral rights for the publications made accessible in the public portal are retained by the authors and/or other copyright owners and it is a condition of accessing publications that users recognise and abide by the legal requirements associated with these rights.

- Users may download and print one copy of any publication from the public portal for the purpose of private study or research.

- You may not further distribute the material or use it for any profit-making activity or commercial gain

- You may freely distribute the URL identifying the publication in the public portal 


\title{
Is Travel Demand Actually Deep? An Application in Event Areas Using Semantic Information
}

\author{
${\text { Ioulia } \text { Markou }^{\circledR} \text {, Filipe Rodrigues }}^{\circledR}$, and Francisco C. Pereira
}

\begin{abstract}
In transportation, nature, economy, environment, and many other settings, there are multiple simultaneous phenomena happening that are of interest to model and predict. Over the last few years, the traffic data that we have at our disposal have significantly increased, and we have truly entered the era of big data for transportation. Most existing travel demand prediction methods mainly focus on capturing recurrent mobility trends that relate to habitual/routine behavior, and on exploiting short-term correlations with recent observation patterns. However, valuable information that is often available in the form of unstructured data is neglected when attempting to improve forecasting results. Particularly, under non-recurrent conditions, such as large events, or incidents, we need much better models. In this paper, we explore time-series data and semantic information combinations using machine learning and deep learning techniques in the context of creating a prediction model that is able to capture in real-time future stressful situations of the studied transportation system. We apply the proposed approaches in event areas in New York using publicly available taxi data. We empirically show that the proposed models are able to significantly reduce the error in the forecasts. The importance of semantic information is highlighted in all presented methods and the final mean absolute error of our prediction is decreased by $\mathbf{2 3 . 8 \%}$ for a three months testing period.
\end{abstract}

Index Terms-Time series forecasting, taxi demand, special events, semantic information, topic modeling, deep learning, deep Gaussian processes.

\section{INTRODUCTION}

A LONG with the evolution of the Internet, the information contributed publicly by all of us keeps increasing substantially. Through popular websites and social platforms such as Facebook, Twitter, Wikipedia etc. that we frequently visit and update, it is possible nowadays to collect information about popular events that happened in the past as well as information for those planned in the near future. In most cases, this information is typically in the form of unstructured natural-language text. Nevertheless, using screen scraping techniques or Application Programming Interfaces (APIs) we are able to retrieve accurate spatio-temporal details that define

Manuscript received June 18, 2018; revised October 9, 2018 and December 17, 2018; accepted January 31, 2019. This work was supported in part by the People Programme (Marie Curie Actions) of the European Union's Seventh Framework Programme (FP7/2007-2013) under REA Grant Agreement 609405 (COFUNDPostdocDTU), and in part by the European Union's Horizon 2020 Research and Innovation Programme under the Marie Sklodowska-Curie Individual Fellowship H2020-MSCA-IF2016, ID 745673. The Associate Editor for this paper was J. M. Alvarez. (Corresponding author: Ioulia Markou.)

The authors are with the Department of Management Engineering, Technical University of Denmark, DK-2800 Kgs. Lyngby, Denmark (e-mail: markou@dtu.dk; rodr@dtu.dk; camara@dtu.dk).

Digital Object Identifier 10.1109/TITS.2019.2897341 a special event, such as its title (what), starting and ending time (when), and venue (where) in the area of our interest. This information may prove to be reasonably valuable for the understanding of observed phenomena in a transport system that are directly related to scheduled events and to the foresight of similar situations in the future.

Disruptions due to special events are a well-known challenge in transport operations, since the transport system is typically designed for habitual demand. Large events (e.g. music concerts, sport games, political rallies) do not receive any special treatment or attention, which often creates non-recurring congestion and overcrowding. Taxi-calling platforms, such as Uber [1], Grab [2] and Beat [3] are becoming increasingly popular, especially in situations of traffic congestion, because they can efficiently facilitate resource allocation. Through their application, passengers are able to call or pre-order a taxi, even when they are located in an area where it is very hard to find a driver. This trend, therefore, proves that there is a tremendous need for better taxi fleet organization and taxi distribution from a taxi center, according to the demand of an entire city [4].

The prediction of taxi demand is challenging because it is correlated with many parameters of underlying information. Currently, the general practice is to rely on formal processes and manual work. For very big events, such as the Olympic games or football world cup matches, the event organizers engage with operators and authorities to meet the enormous demand. For smaller events though, this task is labour-intensive and even with a list of events, their impact is hard to estimate. A timely and accurate notion of demand impact is accordingly needed in order to design adequate system changes and to disseminate appropriate information to the public.

One of the main contributions of this paper is the development of a real-time demand prediction model that is able to forecast taxi demand using special events' data around venues. The time window of the study comprises 4 years (2013-2016) and our work is focused on New York City (NYC). Using a large-scale public dataset of 1.1 billion taxi trips and event data from the Web, we empirically show the value of modeling textual information associated with the events, and that the proposed machine learning approaches are able to outperform other methods from the state of the art by combining information from different sources and formats.

In the context of new forecasting models formulation, the use of tools gaining ground day by day has also been attempted. More specifically, along with the machine learning techniques we will explore deep learning architectures that over the last decade have made major advances in solv- 
ing artificial intelligence problems in different domains such as speech recognition, visual object recognition, and video processing [5]. It is precisely this success of deep learning in handling different types of data, such as images, audio and text from different domains, that makes it particularly wellsuited for the data fusion problem of combining time-series and textual data.

The remainder of this paper is structured as follows. A literature review on prediction applications and topic modeling is presented next. Then, we describe our datasets and case study (Section 3) and present our model (Section 4). The paper ends with the conclusions (Section 5).

\section{LiterATURE REVIEW}

The available techniques for demand forecasting, the accessible spatial datasets, as well as the potential of events information and topic modeling for transportation problems, should be taken into consideration for an accurate demand prediction model formulation.

\section{A. Prediction Applications Using Taxi Data}

In urban systems, nature, economy, environment, and many other settings, there are multiple simultaneous phenomena happening that are of interest to model and predict. Taxi demand prediction is one of the non-trivial research subjects that attracts particular interest due to its inherent complexity. Taxi centers need to better organize their fleet, so that they can maximize their profits, as well as the satisfaction of their employees and customers. Therefore, a better demand prediction can be beneficial for all interested parties. It should be also noted that a good prediction model will be necessary in the future, because self-driving taxis will need to decide where to roam before picking up passengers.

Several methods have been proposed to predict taxi demand, including probabilistic models [6], neural networks [7] and time series modeling [8], [9]. A unified linear regression model that outperforms other popular non-linear models in the prediction accuracy of the Unit Original Taxi Demand (UOTD) is proposed by Tong et al. [10]. A simple model structure that eliminates the need for repeated model redesign proves to be able to behave better in prediction scenarios with highdimensional features.

One of the main pillars of research using taxi data is the modeling of the dispatching center. Zhang et al. [11] propose a taxi drivers' recommendation system which is based on the combination of drivers' location and demand hotspot's hotness. They focus on the travel requirements' understanding as well as on the reduction of cruising time and wasted energy. Miao et al. [12] propose a dispatching framework for balancing taxi supply in a city. Their objectives include matching spatio-temporal ratio between demand and supply for service quality with minimum current and anticipated future taxi idle driving distance.

New York City has been a subject of several studies, since its yellow and green taxi public dataset is easily accessible and sufficiently detailed. Markou et al. [13] used kernel density analysis for demand fluctuations detection and analysis. Significant deviations from the average day were correlated with disruptive event scenarios such as extreme weather conditions, public holidays, religious festivities, and parades. Morgul and Ozbay [14] present an empirical assessment of taxicab drivers labor supply. Yang and Gonzales [15] identify locations and times of day where there is a mismatch between the availability of taxicabs and taxi service demand. Zhao et al. [16] use entropy and the temporal correlation of human mobility to measure the demand uncertainty at the building block level. They implemented three prediction algorithms to validate their maximum predictability theory. Some other research studies used this taxicab data to explore taxicab drivers airport pick-up decisions [17], or travel time variability analysis [18].

\section{B. Deep Models in Transportation}

Deep learning is evolving rapidly in solving problems that have resisted the best attempts of the artificial intelligence community for many years. It has proven to be able to find intricate structures in high-dimensional data, and thus it is an important tool in various applications in the domain of science [19]. In the field of transportation and urban mobility there are already studies showing deep learning's successfullness.

Lv et al. [20] proposed a deep-learning-based traffic flow prediction method that takes into consideration the traffic flow features as learned by a stacked autoencoder model (SAE). Results comparison with more traditional approaches based on Support Vector Regression (SVR) and radial basis functions (RBFs) showed proposed method's superiority. Ma et al. [21] proposed a long short-term memory neural (LSTM) network for travel speed prediction. Their empirical results on data from Beijing indicate that LSTMs outperform other methods such ARIMA and SVR, which the authors justify with the ability of LSTMs to capture long-term dependencies over the time-series. A model with Mixture Density Networks (MDN) on top of LSTM was proposed by $\mathrm{Xu}$ et al. [7]. In their approach, the city is previously divided in smaller areas and then the LSTM-based model is used to jointly predict the taxi demand for the next time-step in all the areas. Finally, the prediction of crowds' traffic in city's regions using a deeplearning based approach, called ST-ResNet, is presented by Zhang et al. [22]. Experiments on two types of crowd flows in Beijing and New York City (NYC) demonstrate that the proposed method outperforms standard approaches such as ARIMA and vector auto-regressive models.

While the approaches described above demonstrate the potential of deep learning for transportation problems, none of these approaches consider the effect of events in order to improve their predictions. The deep learning approaches proposed in this paper aim at bridging this gap by focusing on event areas and showing that data fusion techniques that combine text data about events and time-series observations of mobility demand can significantly improve predictions.

Finally, in addition to the methodologies presented above, this research gives a further insight into another deep framework presented by Damianou and Laurence [23], the deep Gaussian process models. Gaussian processes (GPs) are already a well-known technique for their flexibility and their 
non-parametric, probabilistic approaches to function estimation in an analytically tractable manner. In the deep GP framework, the data is modeled as the output of a multivariate GP whose inputs are governed by another GP. Experiments with simulated and real data showed that always DGPs exceed or match the performance of a single layer GP. Salimbeni and Deisenroth [24] have also shown that the DGPs often exceed the single layer significantly, even when the quality of the approximation to the single layer is improved. For mobility trends where we have two basic components, namely the habitual behaviour of drivers and their provisional behavior which comprises the human need for entertainment, social interaction and public expression, we believe that DGPs can provide the tools for capturing this multi-factorial nature. To the best of our knowledge they have not been tested yet as a traffic prediction method, and we will try to investigate their utility through this research.

\section{Internet as a Data Source for Special Events}

Internet, and more specifically the several social networking services that exist, has become a popular distribution outlet for users looking to share their experiences and interests on the Web. Taking as an example the Facebook, which has over 1.86 billion monthly active Facebook users (Facebook MAUs) worldwide, it is clearly understood that the information derived from the above platforms, can undeniably help discerning explanations about observed real-world phenomena, such as non-habitual overcrowding scenarios.

Due to the importance of special events impact in urban mobility, it is not surprising that they are a predominant part of transportation research. Fortunately, the Internet is rich in information about public special events. In an earlier work, using public transport data Pereira et al. [25] compared an origin/destination (OD) prediction model with and without simple information obtained from the Internet, such as event type or whether the performer/event had a Wikipedia page. It was verified that such information could reduce the root mean squared error (RMSE) by more than 50\% in each OD. In another study, Pereira et al. [26] presented a machine learning model that classifies aggregated crowd observations into explanatory components. After the identification of overcrowding hotspots in the city-state of Singapore, potential explanations from several event announcements websites were retrieved. It was observed that the model is able to recover observed total impacts with an RMSE between $55 \%$ and $85 \%$.

The internet is also a valuable source for other aspects of mobility research. For example, Twitter has been used for crisis management [27], [28], urban management and planning [29], the analysis of different aspects of mobility [30] and the mobility characteristics of different nations [31]. Due to the complexity of the exploration of the open Web (e.g. using Google search), the use of internet data in transportation, however, is currently limited to manually defined sources and highly fine-tuned processes.

\section{Topic Models}

A considerable amount of important information about a planned event is in textual form. Adding to other structured information, such as date, time and location, we can find useful details concerning its content in the description, title, comments on the website hosting the announcement. To obtain an automated system, we still need to convert such data into a proper representation that a machine learning can understand. However, the dimensionality of the machine learning model will be increased beyond reasonable if we explicitly include the text, word by word. Natural language is rich in synonymy and polysemy, different announcers and locations may use different words, besides it is not always obvious which words are more "relevant". Topic modeling is the research topic that focuses on covering these weaknesses.

The approach of topic modeling is to represent a text document as a finite set of topics. These topics correspond to sets of words that tend to co-occur together rather than a single word associated with a specific topic. For example, a rock festival textual description could have a weight $w_{1}$ assigned to topic 1 (e.g. words related to concerts in general), $w_{2}$ of topic 2 (e.g. words related to festivals), $w_{3}$ of topic 3 (e.g. words related to the venue descriptions) and so on. In particular, we use a specific technique that is called Latent Dirichlet Allocation (LDA). For the readers that are familiar with Principal Components Analysis (PCA), there is a simple analogy: PCA re-represents a signal as a linear combination of its eigenvectors, while LDA re-represents a text as a linear combination of topics. In this way, we reduce the dimensionality from the total number of different words of a text to the number of topics, typically very low. Each document is represented as a distribution over topics, and each topic is a distribution over words. For further details concerning LDA's generative process please refer to the original article of David Blei and colleagues [32].

In the particular domain of urban computing, Pereira et al. [33] studied the problem of using event data to help predict public transport demand. Their approach consists of using LDA to learn a topic model, and using the topic assignments as features in a shallow neural network model.

Other recent research efforts have focused on tools dealing with event identification in real time using micro-blog services. Kireyev et al. [34] suggest that the family of topics models is a particularly promising tool for disaster response agencies, as Twitter often provides critical up-to-date and on-location updates about an unfolding crisis. Ramage et al. [35] present a partially supervised learning model (Labeled LDA) that maps the content of the Twitter feed into different dimensions, including substance, style, status, and social characteristics of posts. Ma et al. [36] present a scalable implementation of a topic modeling (Adaptive Link-IPLSA) based method for online event analysis, which summarize the gist of the massive amount of changing tweets. All these studies further confirm the practicability of topic modeling for microblog representation.

While the approaches described above demonstrate the potential of events information and topic modeling for transportation problems, none of them work on a real-time basis. The machine learning techniques proposed in this paper take into consideration the advantage of short-term time-series 


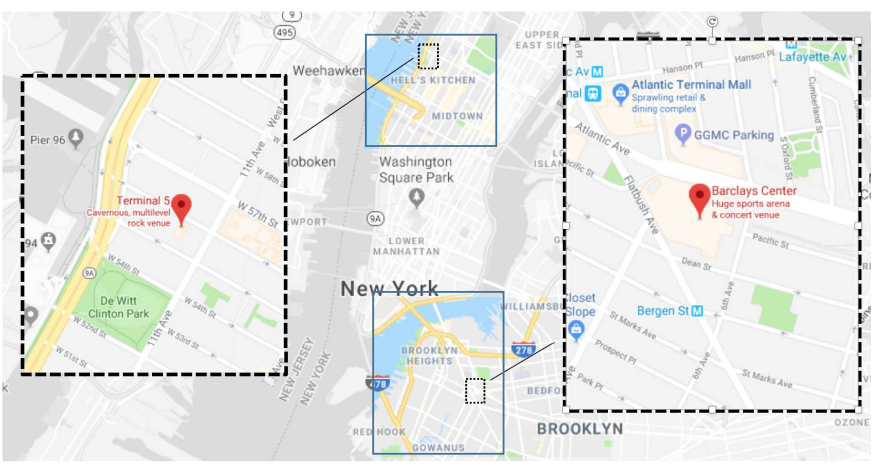

Fig. 1. Map of the two study areas.

trends and predict taxi demand having knowledge of traffic conditions in the near past. The developed model can be used to instantaneously inform interested parties about possible increased demand around venues and better critical fleet distribution for system requirements fulfillment.

\section{DATA DESCRIPTION AND PREPARATION}

In this research, we work with two major datasets: events and taxi data. The latter is distributed by technology providers of authorized under the Taxicab \& Livery Passenger Enhancement Programs (TPEP/LPEP) and were made publicly available by the NYC Taxi and Limousine Commission (TLC) [37]. We use taxi data from 1/1/2013 through 6/30/2016, which includes around 600 millions taxi trips after data filtering. The dataset specifies for each drop-off and pick-up event the GPS location and the time-stamp.

Based on this data, we looked at a list of the top venues in NYC [38] and selected the two venues for which more complete event records were available online: the Barclays Center and Terminal 5. The first venue is located in the heart of Brooklyn and it is the state-of-the-art home of the NBAs Brooklyn Nets and the NHLs New York Islanders. It is one of the most popular facilities in the New York metropolitan area because it hosts many sold-out concerts, conventions and other sporting and entertainment events. It is ranked top five globally in 2015 for gross revenue and attendance by Billboard and Venues Today. On the other hand, the Terminal 5 is a 3 -floor venue that regularly hosts concerts with many different audiences and that is located in the heart of Manhattan. Given the geographical coordinates of these two venues, we selected all the taxi pickups that took place within a bounding box of \pm 0.003 decimal degrees (roughly 500 meters) to be our study areas. Figure 1 shows a map of these areas.

Regarding the event data, it was extracted automatically from the Web using either screen scraping techniques or APIs. For the Barclays Center, the event information was scrapped from its official website, since it maintains a very accurate and detailed calendar. We collected a total of 751 events since its inauguration in late 2012 until June 2016. As for the Terminal 5, we used the Facebook API to extract 315 events for a similar time period. In both cases, the event data includes event's title, date, time and description.

\section{A. Preliminary Analysis of Time-Series Data}

The raw dataset that we obtained from TLC [37] includes fields capturing pick-up and drop-off dates/times, pick-up and drop-off locations, trip distances, itemized fares, rate types, payment types, and driver-reported passenger counts. Within the framework of the forecasting model that we want to formulate for Barclays Center and Terminal 5, the filtered taxi trip records are additionally aggregated by hour.

For most regions, the taxi demand is governed by a certain amount of randomness (e.g., unexpected events) and some degree of regularity (e.g., weekly patterns), which can be exploited for prediction. Before the configuration of initial modeling structure, we decided to remove any deterministic trends and focus our analysis on the remaining fluctuations. A simple, yet very effective way, of identifying these daily or weekly recurring patterns is by constructing a historical averages model, which computes the individual averages for each (hour of day, day of the week) pair based on historical data (from the train set only). The historical averages then represent a fixed recurring trend, which can be easily removed from the data.

Based on our experience with various time-series forecasting problems with urban mobility data, by removing the burden of capturing these well-known recurrent trends from the model, this simple detrending procedure significantly improves the predictive performance. With our focus on the demand component that is directly correlated with events scheduled on that day, we managed to see more accurate predictions. In fact, as our experiments demonstrate, it makes even the simplest linear models quite competitive baselines to outperform.

\section{B. Text Data Pre-Processing}

Generally, textual data mined from the Web is difficult to process in its original state. Specific pre-processing steps are usually required in order to make it more amenable to learning methods, and more specifically to the topic modelling stage that will follow. Therefore, we follow a simple conventional text-processing pipeline consisting of:

- HTML tag removal

- Lowercase transformation for words' variability restriction purposes

- Tokenization, a tool that divides a sequence of characters into pieces of tokens

- Lemmatization for inflectional endings removal, and words return to their base form (lemma)

- Stopwords and very frequent words removal, which typically do not bring any additional useful information

- Removal of words that appear only once in the whole dataset

\section{Demand Prediction Model Formulation}

Our prediction model should demonstrate the hypothesis that contextual information is significant for real-time taxi demand prediction in the vicinity of special event venues. Generalizing to other cases, and upon available event data, this should be valid to any area in which demand can be somehow associated with available contextual information, 
as for example school areas and information about school holidays, shopping areas and large sales, and main governmental buildings and public demonstrations.

\section{A. Initial Model Selection and Comparison}

The proposed approach is focused on hourly short-term predictions. The specific time-step was chosen, as it represents the average time that a driver needs to cover the maximum distance within Manhattan. Consequently, demand predictions for the following hour can be very beneficial for a taxi company or a share-mobility application that aims at optimizing its fleet exploitation.

For the implementation of our short-term predictions we consider first two simple approaches (i) a linear regression (LR) model and (ii) a Gaussian process (GP) regression model using the scikit-learn machine learning library in Python [39]. The former is chosen for its simplicity and interpretability and the latter because it is flexible enough to represent a wide variety of interesting model structures. GPs have shown to achieve state-of-the-art results for various tasks, such as traveltime prediction [40], traffic volume forecasting [41] and public transportation trips predictions around special event areas [42].

For models' performance validation and comparison we will use the mean absolute error (MAE), the root mean square error (RMSE) and the coefficient of determination $\left(R^{2}\right)$, computed as follows:

$$
\begin{aligned}
M A E & =\frac{1}{N} \sum_{n=1}^{N}\left|y_{n}-\hat{y}_{n}\right| \\
R M S E & =\sqrt{\frac{1}{N} \sum_{n=1}^{N}\left(y_{n}-\hat{y}_{n}\right)^{2}} \\
R^{2} & =1-\frac{\sum_{n=1}^{N}\left(y_{n}-\hat{y}_{n}\right)^{2}}{\sum_{n=1}^{N}\left(y_{n}-\bar{y}\right)^{2}}
\end{aligned}
$$

where $N$ denotes the number of instances in the dataset, $\hat{y}_{n}$ is the predicted taxi pick-ups count for the $n_{t h}$ instance, $y_{n}$ is the corresponding true pick-ups count and $\bar{y}$ is the mean of the observed counts.

\section{B. Model Formulation}

The final hourly taxi pick-ups and drop-offs will be used for the establishment of our first two basic models: an elemental model with only pick-ups information; and a second model with both pick-up and drop-off information. For the initial models' formulation and evaluation we focused on Barclays Center.

The starting point of our forecasting model is to use a $12^{\text {th }}$ Order Autoregressive Model, namely $Y_{t}$ is regressed against $Y_{t-1}, Y_{t-2}, \ldots, Y_{t-11}$, where $Y$ represents the detrended taxi pickups counts.

$$
\hat{Y}_{t+1}=\hat{\beta}_{0}+\hat{\beta}_{1} Y_{t}+\ldots+\hat{\beta_{12}} Y_{t-11}
$$

where $\hat{\beta}_{0}, \hat{\beta}_{1}, \ldots, \hat{\beta_{12}}$ are estimated using data through period $t$. The order of the autoregressive model was chosen after several experiments with different number of lags. The best performance was achieved using information from the previous 12 hours only.

The first three years of our dataset (2013-2015) are model's training set and the first six months of 2016 (January 2016 June 2016) our test set. The results of this simple model form the baseline of our analysis (see Table III).

Then, taxi drop-off counts were introduced. We insert the same number of detrended drop-off counts, thus increasing the number of model's dependent parameters to 24 .

$$
\begin{aligned}
\hat{Y}_{t+1}=\hat{\beta_{0}}+\hat{\beta_{1,1} Y_{t}}+\ldots+\hat{\beta_{1,12} Y_{t-11}} \\
+\hat{\beta_{2,1} D_{t}}+\ldots+\hat{\beta_{2,12} D_{t-11}}
\end{aligned}
$$

where $D$ represents the detrended hourly drop-off counts.

The performance of our enriched model is significantly improved (Table III). The $R^{2}$ score has increased by almost $7 \%$, thus demonstrating how important it is to introduce information about taxi arrivals at the same area, at an earlier time. Through a sensitivity analysis of models' parameters, it was also found that the more recent the pickup lag is, the greater its significance to the final model performance. The final parameter vector $\beta=\left(\beta_{0}, \beta_{1,1}, \ldots, \beta_{2,12}\right)$ showed us that the drop-off lags $D_{t-3}$ and $D_{t-4}$ appear to contribute more effectively than the rest of drop-off lags. This result allows us to understand that there is a strong correlation between taxi drop-offs three to four hours before our calculated pickups; a conclusion that seems to be related ultimately to the average duration of an event that Barclays Center hosts, such as a basketball game or a concert.

\section{Weather Data}

Before including information about events that took place in the selected venues, we decided to evaluate the contribution of weather data in the hourly demand prediction. The dataset was obtained from the National Oceanic and Atmospheric Administration (NOAA) and corresponds to daily observations from a weather station located in the Central Park in NYC. It contains information about daily minimum and maximum temperatures, daily precipitation, wind, presence of snow, fog, snow depth, etc.. The performance of the enhanced model showed us that weather does not contribute positively to our forecast's accuracy. All three error statistics remain almost unaffected (see Table III). This result may be due to the fact that we have only daily weather information at our disposal and not for shorter time periods. Therefore, for an hourly demand prediction model, information about the weather of an entire day is probably too aggregated. Additionally, our model can "capture" changes in demand and supply due to weather conditions indirectly from the pick-up and drop-off lags. If extreme weather conditions, such as a blizzard or a thunderstorm, occur on a particular day, then the reduced taxi demand in the previous hour will reflect this phenomenon into our model.

\section{Event Information}

The selected area around Barclays Center shows significant changes in taxi demand mainly after afternoon hours. 


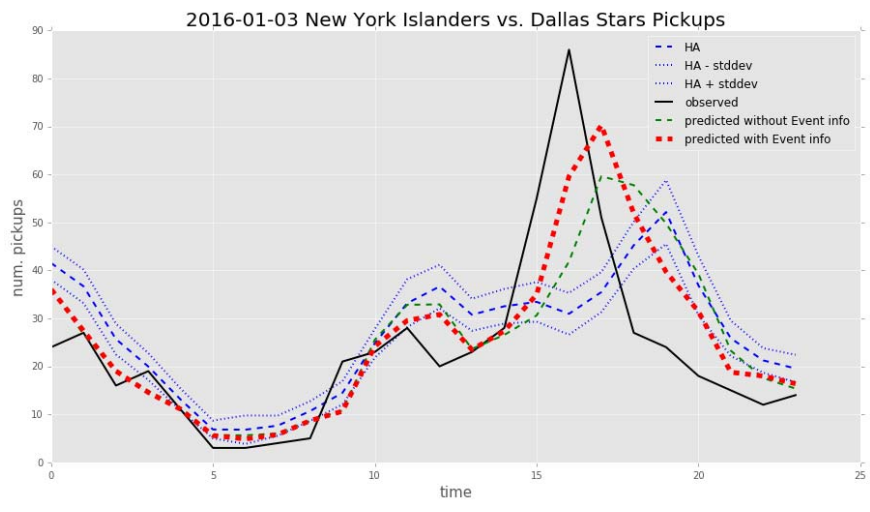

Fig. 2. Prediction results for an event day using only taxi data (green line) and event information (red thicker line).

The cause of most of these intense fluctuations is possibly several events that take place in this popular venue. As mentioned in Section III, the events' dataset includes all records' details that could be scrapped from the official Barclays's Center website; title, date, and event description. Utilizing the above information, we choose to introduce into our enhanced forecasting model six new parameters that indicate the time position of each hourly pick-up count record in relation to the start time of an event.

More specifically, our new parameters are binary identifiers that indicate if there is an event "3 hours before", "2 hours before", "1 hour before", "1 hour after", "2 hours after" or "3 hours after" the current dataset instance. The start time of venue's events and the date-time information of each entry were used to complete the identifiers' values.

The structure of the updated linear regression model is:

$$
\begin{aligned}
& \hat{Y}_{t+1}=\hat{\beta_{0}}+\hat{\beta_{1,1}} Y_{t}+\ldots+\hat{\beta_{1,12}} Y_{t-12} \\
& +\hat{\beta_{2,1} D_{t}}+\ldots+\hat{\beta_{2,12}} D_{t-12} \\
& +\hat{\beta_{3,1} E_{\text {Event }} 3 h_{-} \text {bef }, t}+\ldots+\hat{\beta_{3,6} \text { Event }_{3 h \_a f t e r, t}}
\end{aligned}
$$

where Event $3 h_{-}$bef, $t$, Event $_{3 h_{-} \_ \text {ffter, } t}$ represent the binary variables described above. We excluded variables related with weather, since their contribution was proven insignificant. The same training and test were used for the evaluation and comparison of the updated model structure (see Table III). The improvement rate is small but important in an already wellbehaved model. The specific parameters allowed our model to predict demand peaks in a more accurate time position. This conclusion was obtained after visualizing the previous and updated model results in daily plots. A characteristic example is presented in Fig. 2. The historical demand average (HA) is represented by the blue dashed line. The hourly predictions of our previous model are depicted with the green dashed line, and the new predictions, enhanced by adding the binary event identifiers, with the red thick dashed line. It is obvious, that the updated values are better placed in time.

\section{E. Introduction of Topics}

In the fourth and final stage of our existing linear regression model optimization we decided to implement a Latent
Dirichlet Allocation (LDA) process on the events' description. LDA assumes the following generative process:

1) Draw a topic $\beta_{k}$ from $\beta_{k} \sim \operatorname{Dirichlet}(\eta)$ for $k=$ $1 \ldots K$

2) For each document $d$ :

a) Draw topics proportions $\theta_{d}$ such that $\theta_{d} \sim$ Dirichlet $(\alpha)$

b) For each word $w_{d, n}$ :

i) Draw topic assignment $z_{d, n} \sim \operatorname{Multinomial}\left(\theta_{d}\right)$

ii) Draw word $w_{d, n} \sim \operatorname{Multinomial}\left(\beta_{z_{d, n}}\right)$

The parameters $\alpha$ and $\eta$ are hyperparameters that indicate respectively the priors on per-document topic distribution and per-topic word distribution, respectively. Thus, $w_{d, n}$ are the only observable variables, all the others are latent in this model. For a set of $D$ documents, given the parameters $\alpha$ and $\eta$, the joint distribution of a topic mixture $\theta$, word-topic mixtures $\beta$, topics $z$, and a set of $N$ words is given by:

$$
\begin{aligned}
p(\theta, \beta, z, w \mid \alpha, \eta) & =\prod_{k=1}^{K} p\left(\beta_{k} \mid n\right) \prod_{d=1}^{D} p\left(\theta_{d} \mid a\right) \\
& =\prod_{n=1}^{N}\left(p\left(z_{d, n} \mid \theta_{d}\right) p\left(w_{d, n} \mid \beta_{k}, k=z_{d, n}\right)\right)
\end{aligned}
$$

Broadly speaking, the training task is to find the posterior distribution of the latent variables (the per-document topic proportions $\theta_{d}$, the per-word topic assignments $z_{d, n}$ and the topics $\beta_{k}$ ) that maximize this probability.

The parameter that we mainly focused in this study is the number of topics. We tested a range of values between 5 and 30 , and we empirically concluded that the value of 10 yielded the best model results. With 10 topics we are able to capture all kinds of events included in our event database, and we also narrow down possible equivocal topics that could deteriorate our results. The other parameters, the $\alpha$ and $\eta$ priors, were kept as default (1.0/(number of topics)). To confirm this was a safe choice, we ran several iterations with different initial $\alpha$ and $\eta$ priors and they generally converged to similar outcomes. The LDA results are presented in Table I.

Each event record now corresponds to a unique topic assignment, namely a vector of 10 values. Building on the previous 6 binary event identifiers, and for each hourly taxi demand count we added the topic assignment of the event that was planned "3 hours before", "2 hours before", "1 hour before", "1 hour after", "2 hours after" or "3 hours after". Therefore, each aggregated taxi record is enriched with $6 * 10$ new variables, where 6 is the number of the previously defined event identifiers.

For example, if the taxi pick-up count refers to $6 \mathrm{pm}$ and from our event dataset we know that there will be an event at $8 \mathrm{pm}$, whose topic assignment vector is $[0,0,0.87,0,0.01,0.05,0,0,0.07,0]$ then the 60 new variables that our model is going to receive are shown in Table II.

We provide these apparently redundant features in the context of experimenting with the best model representation and thus with our final best predictor. Through the implementation of many tests, it was found that the optimal performance of the model is achieved by introducing only the last 30 parameters 
TABLE I

LDA RESULTS

\begin{tabular}{|c|c|c|}
\hline Topic & No. Events with $\theta_{d}>0.8$ & Popular Words \\
\hline \hline Topic_1 & 24 & ice, disney, present, magic, new \\
\hline Topic_2 & 72 & basketball, championship, atlantic, game, tournament \\
\hline Topic_3 & 10 & show, artist, box, office, special \\
\hline Topic_4 & 32 & music, atlantic, championship, basketball, game \\
\hline Topic_5 & 22 & game, marriot, corporate, bridge, hotel \\
\hline Topic_6 & 10 & train, service, islander, view, time \\
\hline Topic_7 & 34 & tour, album, show, meet, up \\
\hline Topic_8 & 12 & circus, family, out, space, earth \\
\hline Topic_9 & 12 & dinner, reservation, jay, menu, restaurant \\
\hline Topic_10 & 42 & champion, game, group, boxing, hoop \\
\hline
\end{tabular}

TABLE II

EXAMPLE OF TOPIC MODELING INTRODUCTION

\begin{tabular}{|c|c|c|}
\hline Event identifier & Value [0 or 1$]$ & Topic Variables Assignment \\
\hline 3 hours before & 0 & {$[0,0,0,0,0,0,0,0,0,0]$} \\
\hline 2 hours before & 0 & {$[0,0,0,0,0,0,0,0,0,0]$} \\
\hline 1 hour before & 0 & {$[0,0,0,0,0,0,0,0,0,0$} \\
\hline 1 hour after & 0 & {$[0,0,0,0,0,0,0,0,0,0]$} \\
\hline 2 hours after & 1 & {$[0,0,0.87,0,0.01,0.05,0,0,0.07,0]$} \\
\hline 3 hours after & 0 & {$[0,0,0,0,0,0,0,0,0,0]$} \\
\hline
\end{tabular}

TABLE III

Linear Regression Results - 6 Months Period

\begin{tabular}{|c||c|c|c|}
\hline Input Data & MAE & RMSE & $R^{2}$ \\
\hline \hline Pick-up Lags & 9.733 & 19.073 & 0.750 \\
\hline Pick-up \& Drop-off Lags & 8.772 & 16.298 & 0.817 \\
\hline Pick-up \& Drop-off Lags \& Weather & 8.824 & 16.379 & 0.815 \\
\hline $\begin{array}{c}\text { Pick-up \& Drop-off Lags \& } \\
\text { Event info }\end{array}$ & 8.689 & 16.024 & 0.823 \\
\hline $\begin{array}{c}\text { Pick-up \& Drop-off Lags \& } \\
\text { Event info \& Topics }\end{array}$ & 8.301 & 14.932 & 0.847 \\
\hline
\end{tabular}

that correspond to the time period after event's start time, namely the identifiers "1 hour after", "2 hours after" and "3 hours after". This is probably justified by the fact that our model already contains information about the time window before the start of the event (by using drop-off lags) and not sufficient information for the time period after its start time. Our model is considerably improved, and the updated values of MAE, RMSE, and $R^{2}$ prove it (see Table III).

\section{F. Model Evaluation Using Only Event Days}

To summarize, from the previous stage of our analysis, four models were developed:

- The baseline model using only pick-up lags

- The pick-up - drop-off model which is enriched with drop-off lags

- The event identifier model, where six binary event identifiers were introduced

- The topic model with additional 30 topic variables based on LDA's results.

Each model is an enhanced version of the previous one. The proposed architecture performs respectively well on event days. Table IV summarizes those results. The exploitation of drop-off parameters improves demand predictions by $10.11 \%$.
TABLE IV

Linear Regression Results - Event Period

\begin{tabular}{|c||c|c|c|}
\hline Input Data & MAE & RMSE & $R^{2}$ \\
\hline \hline Pick-up Lags & 11.037 & 22.480 & 0.742 \\
\hline Pick-up \& Drop-off Lags & 9.840 & 18.939 & 0.817 \\
\hline Pick-up \& Drop-off Lags \& Weather & 9.819 & 18.923 & 0.817 \\
\hline $\begin{array}{c}\text { Pick-up \& Drop-off Lags \& } \\
\text { Event info }\end{array}$ & 9.597 & 18.230 & 0.830 \\
\hline $\begin{array}{c}\text { Pick-up \& Drop-off Lags \& } \\
\text { Event info \& Topics }\end{array}$ & 8.585 & 15.837 & 0.872 \\
\hline
\end{tabular}

This significant improvement proves once again the importance of monitoring traffic network's conditions before an event starts. The dummy variables of the third model have small but positive effect on the final result, while topics' impact is once again significant (5\% further improvement on an already fairly reliable model).

\section{G. Gaussian Processes for Demand Prediction}

Besides these baselines, the proposed approach is further compared with another popular method from the state of the art for time-series forecasting, the Gaussian processes (GPs) [43]. We chose new independent training, validation and test sets, since GPs implementation is a computational demanding process. The training set consists of 6 months of observations (January - June 2015), the validation set contains the first two months of 2016 (January-February) and the remaining four months of the first half of 2016 were used for testing. The hyper-parameters of GPs, namely the lengthscale parameter of the Radial-basis function (RBF) kernel, and the Tikhonov regularization of the assumed covariance between the training points (alpha) [44], were tuned based on their performance on the validation set. An exhaustive search procedure of all possible combinations within a predefined set of possible value parameters was used.

Along with GPs implementation, the corresponding forecasts of linear regression models were also evaluated. Both machine learning techniques use the same time intervals for training and testing. Furthermore, more experiments were implemented using Terminal 5's datasets. The final results are presented in Table V.

There are several very interesting aspects that are worth discussing. From the first three models, it is concluded that the performance of LR and GPs is similar. A remarkable change 
TABLE V

LR AND GP COMPARISON - 4 MONTHS PERIOD

\begin{tabular}{|c|c|c|c|c|c|c|c|c|c|c|c|c|}
\hline \multirow[b]{3}{*}{ Input Data } & \multicolumn{6}{|c|}{ Barclays Center } & \multicolumn{6}{|c|}{ Terminal 5} \\
\hline & \multicolumn{3}{|c|}{ Linear Regression } & \multicolumn{3}{|c|}{ Gaussian Processes } & \multicolumn{3}{|c|}{ Linear Regression } & \multicolumn{3}{|c|}{ Gaussian Processes } \\
\hline & MAE & RMSE & $R^{2}$ & MAE & RMSE & $R^{2}$ & MAE & RMSE & $R^{2}$ & MAE & RMSE & $R^{2}$ \\
\hline Pick-up Lags & 9.523 & 18.733 & 0.756 & 9.522 & 18.675 & 0.757 & 10.606 & 19.215 & 0.750 & 10.771 & 18.905 & 0.758 \\
\hline Pick-up \& Drop-off Lags & 8.620 & 16.280 & 0.815 & 8.669 & 16.378 & 0.813 & 8.928 & 15.409 & 0.840 & 9.095 & 15.452 & 0.839 \\
\hline $\begin{array}{l}\text { Pick-up \& Drop-off Lags \& } \\
\text { Weather }\end{array}$ & 8.623 & 16.289 & 0.815 & 8.670 & 16.377 & 0.813 & 8.938 & 15.421 & 0.838 & 9.086 & 15.448 & 0.839 \\
\hline $\begin{array}{l}\text { Pick-up \& Drop-off Lags \& } \\
\text { Weather \& Event info }\end{array}$ & 8,577 & 16.369 & 0.813 & 8.571 & 16.396 & 0.813 & 8.828 & 14.969 & 0.849 & 9.070 & 15.380 & 0.840 \\
\hline $\begin{array}{c}\text { Pick-up \& Drop-off Lags \& } \\
\text { Weather \& Event info \& Topics }\end{array}$ & 8.258 & 15.656 & 0.829 & 8.100 & 14.979 & 0.844 & 8.935 & 15.254 & 0.843 & 8.206 & 13.095 & 0.864 \\
\hline
\end{tabular}

TABLE VI

LR AND GP COMPARISON - EVENTS' PERIOD

\begin{tabular}{|c|c|c|c|c|c|c|c|c|c|c|c|c|}
\hline \multirow[b]{3}{*}{ Input Data } & \multicolumn{6}{|c|}{ Barclays Center } & \multicolumn{6}{|c|}{ Terminal 5} \\
\hline & \multicolumn{3}{|c|}{ Linear Regression } & \multicolumn{3}{|c|}{ Gaussian Processes } & \multicolumn{3}{|c|}{ Linear Regression } & \multicolumn{3}{|c|}{ Gaussian Processes } \\
\hline & MAE & RMSE & $R^{2}$ & MAE & RMSE & $R^{2}$ & MAE & RMSE & $R^{2}$ & MAE & RMSE & $R^{2}$ \\
\hline Pick-up Lags & 10.395 & 20.797 & 0.753 & 11.546 & 22.864 & 0.702 & 13.469 & 23.997 & 0.684 & 13.654 & 23.909 & 0.686 \\
\hline Pick-up \& Drop-off Lags & 9.425 & 18.246 & 0.810 & 10.065 & 19.357 & 0.786 & 11.458 & 19.667 & 0.788 & 11.270 & 19.650 & 0.788 \\
\hline $\begin{array}{l}\text { Pick-up \& Drop-off Lags \& } \\
\text { Weather }\end{array}$ & 9.393 & 18 & 0.810 & 9 & 01 & 0. & 80 & 15 & 0.789 & 328 & 19.718 & 0.787 \\
\hline $\begin{array}{l}\text { Pick-up \& Drop-off Lags \& } \\
\text { Weather \& Event info }\end{array}$ & 9.569 & 18.875 & 0.797 & 9.844 & 18.789 & 0.799 & 11.554 & 19.487 & 0.792 & 11.214 & 19.552 & 0.790 \\
\hline $\begin{array}{c}\text { Pick-up \& Drop-off Lags \& } \\
\text { Weather \& Event info \& Topics }\end{array}$ & 9.236 & 18.141 & 0.812 & 8.945 & 17.235 & 0.830 & 11.539 & 462 & 0.793 & 10.912 & 18.996 & 0.799 \\
\hline
\end{tabular}

TABLE VII

Real-Time and Daily Demand Prediction Comparison

\begin{tabular}{|c|c|c|c|c|c|c|c|c|c|c|c|c|}
\hline \multirow[b]{3}{*}{ Input Data } & \multicolumn{6}{|c|}{ Daily Demand Prediction } & \multicolumn{6}{|c|}{ Aggregated Daily Demand Prediction } \\
\hline & & SVR & & Gau & ian Pro & sses & Lin & r Regres & ion & Gau & ian Pro & sses \\
\hline & MAE & RMSE & $R^{2}$ & MAE & RMSE & $R^{2}$ & MAE & RMSE & $R^{2}$ & MAE & RMSE & $R^{2}$ \\
\hline Pick-up \& Drop-off Lags & 120.7 & 167.5 & 0.428 & 145.2 & 199.4 & 0.189 & 48.012 & 61.763 & 0.936 & 71.1 & 102.2 & 0.826 \\
\hline $\begin{array}{c}\text { Pick-up \& Drop-off Lags \& } \\
\text { Weather }\end{array}$ & 120.1 & 166.4 & 0.436 & 127.3 & 176.9 & 0.362 & 48.0 & 61.9 & 0.936 & 70.9 & 102.0 & 0.827 \\
\hline $\begin{array}{l}\text { Pick-up \& Drop-off Lags \& } \\
\text { Weather \& Event info }\end{array}$ & 97.9 & 139.1 & 0.605 & 100.0 & 141.1 & 0.594 & 45.5 & 59.2 & 0.942 & 71.1 & 102.2 & 0.826 \\
\hline
\end{tabular}

appears though with the introduction of topics. For Barclays Center both forecasts are improved, something that does not seem to be confirmed for Terminal 5. More specifically, for the first venue the LR with topics gives a final $R^{2}$ of 0.829 , and an improvement of almost $2 \%$, while for the second venue the final $R^{2}$ is 0.843 , with a deterioration of $0.5 \%$. On the other hand, the GPs seem to respond positively to all imported parameters. With the introduction of topics, the $R^{2}$ is increased by $3 \%$ for Barclays Center and by $2.9 \%$ for Terminal 5 . The GP model emerges as the ideal technique for our real-time forecasting model. Once again, the weather did not make a significant contribution.

The same comparison is implemented using the event periods. Table VI summarizes the final results. GPs prove to be the best performing approach for our predictions. By using topics, the proposed forecasting model reduces its error for both venues. These results clearly highlight how crucial data fusion of time-series data and semantic information can be, in particular for the problem of predicting taxi demand in event areas considered in this paper.

Lastly, from the perspective of transportation practitioners, it is important to note that by exploiting event information automatically extracted from the Web and by developing two regression models for combining this information with historical time-series data, we were able to reduce prediction error in event areas quite dramatically in both study areas. In the case of the Barclays Center area, we started with an initial $R^{2}$ of 0.757 using pick-up lags and we were able to obtain a $R^{2}$ of 0.844 by using the full GP model. Likewise, in the case of the Terminal 5 area, we started with a MAE of 10.77 using pick-up lags and reached a MAE of 8.21 for the full model. These are very significant improvements that emphasize the importance of accounting for the effect of special events when forecasting mobility demand in dynamic and lively urban areas.

Following the performance results presented above, a comparison of our real-time approach with a previous study on daily demand forecasts [45] is implemented and presented in Table VII. The comparison refers to the Barclays Center, and the scores presented in the left part of the table under the title "Daily Demand Prediction", were included as documented in the stated article. GPs were evaluated in both cases, as well as the incorporation of pickups, drop-offs and events' occurrence information.

It is quite obvious that our real-time approach performs significantly better than the daily demand prediction framework. The noteworthy deviation of accuracy is mainly due to the significant enhancement of forecast precision that the hourly 
pick-up and drop-off lags offer to our real-time approach. In those cases where the real-time model was not able to predict a significant change in demand at a particular time, then it will somehow adjust its prediction at the next time-step. Therefore the overall aggregated daily demand forecast will not deviate significantly from the actual value. Additionally, the significance of weather data in the first daily model is noteworthy. As we mentioned earlier, weather information is available only per day and not per hour of the day. Therefore, daily demand and supply fluctuations can be explained, and model's forecast accuracy can be enhanced due to this parameter, since we do not have any other information about the state of the transport system throughout the day (as for the real-time model). Finally, it is worth mentioning that for the daily demand prediction, information about events' occurrence on that particular day plays a decisive role in the accuracy of the model, since we get a better anticipation of the increased demand, while for the real-time model, indications of high demand can be better perceived by the hourly pick-up and drop-off lags.

\section{INCORPORATION OF MORE COMPLEX ARCHITECTURES}

Having previously evaluated our first two simple architectures, we will now consider more complex models: a neural network architecture based on fully-connected (FC) dense layers and a Deep GPs architecture. The motivation for using FC layers results from already existing research studies demonstrating their good performance for certain timeseries forecasting problems [45]-[47]. Deep GPs on the other hand have shown great efficiency on modeling complex data by automatically discovering useful structures and encoding abstract information [23].

\section{A. Deep Gaussian Processes for Demand Prediction}

The architecture of deep Gaussian process that Damianou and Lawrence [23] formulated is presented in Fig. 3. It corresponds to a graphical model with three kinds of nodes: the leaf nodes $Y \in R^{N \times D}$ which are observed, the intermediate latent spaces $X_{h} \in R^{N \times Q_{h}}, h=1, \ldots, H-1$, where $H$ is the number of hidden layers, $Q$ is the number of latent dimensions, and the parent latent node $Z=X_{H} \in R^{N \times Q_{z}}$. In the proposed deep architecture, all intermediate nodes $X_{h}$ act as inputs for the right layer and as outputs for the left layer. So, when we have a simple structure with only two hidden units, the generative method is formulated as follows:

$$
\begin{array}{lll}
y_{n d}=f_{d}^{Y}\left(x_{n}\right)+\epsilon_{n d}^{Y}, & d=1, \ldots, D, & x_{n} \in R^{Q} \\
x_{n q}=f_{q}^{X}\left(z_{n}\right)+\epsilon_{n q}^{X}, & q=1, \ldots, Q, & z_{n} \in R^{Q_{z}}
\end{array}
$$

and the intermediate node is involved in two Gaussian processes, $f^{Y}$ and $f^{X}$, playing the role of an input and an output respectively: $f^{Y} \sim \boldsymbol{G} \boldsymbol{P}\left(0, k^{Y}(X, X)\right)$, and $f^{X} \sim$ $\boldsymbol{G P}\left(0, k^{X}(Z, Z)\right)$. More layers could be added, thus increasing the number of significant model parameters, and system's complexity. For further details concerning Deep GPs' generative process please refer to the original article of Damianou and Lawrence [23].

It has been argued that deep networks have more representational power compared to the simpler/shallow ones.

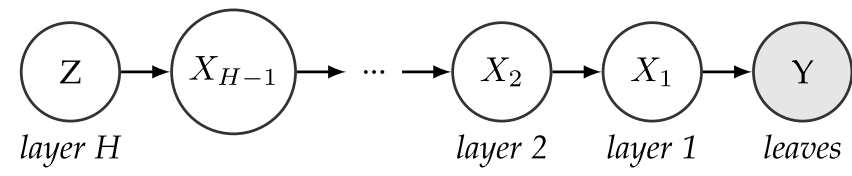

Fig. 3. Representation of a Deep GP model with a cascade of $H$ hidden layers.

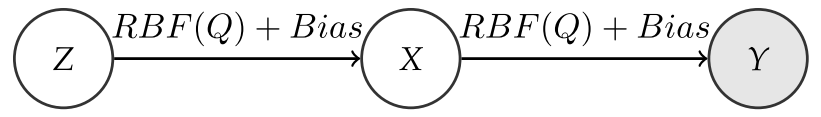

Fig. 4. Implemented Deep GPs architecture.

However, Duvenaud et al. [48] noted that many hidden layers will not always result in a better model. An extreme expression of this effect can result in a pathology and, therefore, very deep networks are not always preferable. In our case, the demand fluctuations do not have very complicated patterns. In the venue areas studied in this case, smooth demand changes are observed during the morning and afternoon hours and in days when we have one or two big events, one or two clear demand peaks are detected (see for example Fig. 2). Therefore, our deep network will not necessarily perform well with many Gaussian processes involved.

After a sufficient number of tests, it is concluded that if our developed model has more than two hidden layers, the prediction results worsened considerably. Therefore, to compare Deep GPs with the previously described model, we developed the architecture depicted in Figure 4. We implemented a simple architecture with two Gaussian processes both with Radial Basis Function kernels in the context of supervised learning. We have a single hidden layer, since the top layer is observed. The kernel length values, as well as the number of latent dimensions of the hidden layer are defined using Bayesian Optimization, a sequential design strategy for global optimization of functions' hyperparameters [49].

We thoroughly studied travel demand predictions for the month of June 2016, since we had distinct demand differences between weeks and we wanted to see how each methodology responds. More specifically, for Terminal 5 we had a week with no events scheduled and a week with four very popular concerts in its calendar. We therefore considered these cases to be representative for the evaluation purposes of this study. The previous 7 months (5000 instances) were chosen as the training set for each method. We wanted to keep the training set's size manageable due to the computational requirements that GPs and subsequently Deep GPs have.

In Tables VIII and IX we summarize the prediction results for a week with no events and four events respectively. In the first case, it is clear that LR and GPs have similar performance. GPs are able to provide acceptable predictions with just the pick-up lags, which highlights their ability to better adapt to fluctuating demand. However, Deep GPs performance is considerably lower than the other two methods. However, by observing their performance in Table VIII, it appears that adding more parameters and subsequently 
TABLE VIII

LR, GPs, DeEP GPS AND DL-FC MOdel Comparison - TERminal 5 - No Events

\begin{tabular}{|c|c|c|c|c|c|c|c|c|c|c|c|c|}
\hline \multirow[b]{2}{*}{ Input Data } & \multicolumn{3}{|c|}{ Linear Regression } & \multicolumn{3}{|c|}{ Gaussian Processes } & \multicolumn{3}{|c|}{ Deep GPs } & \multicolumn{3}{|c|}{ DL-FC } \\
\hline & MAE & RMSE & $R^{2}$ & $\mathrm{MAE}$ & RMSE & $R^{2}$ & MAE & RMSE & $R^{2}$ & MAE & RMSE & $R^{2}$ \\
\hline Pick-up Lags & 10.150 & 13.341 & 0.732 & 7.273 & 10.372 & 0.838 & 10.631 & 13.987 & 0.705 & 7.131 & 9.826 & 0.854 \\
\hline Pick-up \& Drop-off Lags & 6.496 & 8.606 & 0.888 & 6.059 & 8.153 & 0.900 & 9.517 & 12.912 & 0.749 & 6.323 & 8.399 & 0.894 \\
\hline $\begin{array}{c}\text { Pick-up \& Drop-off Lags \& } \\
\text { Weather }\end{array}$ & 6.558 & 8.672 & 0.879 & 6.120 & 8.235 & 0.892 & 9.612 & 13.041 & 0.742 & 6.450 & 8.567 & 0.876 \\
\hline $\begin{array}{l}\text { Pick-up \& Drop-off Lags \& } \\
\text { Weather \& Event info }\end{array}$ & 6.490 & 8.607 & 0.888 & 6.334 & 8.598 & 0.888 & 9.001 & 12.229 & 0.774 & 7.306 & 10.155 & 0.850 \\
\hline $\begin{array}{c}\text { Pick-up \& Drop-off Lags \& } \\
\text { Weather \& Event info \& Topics }\end{array}$ & 6.497 & 8.632 & 0.888 & 6.336 & 8.599 & 0.888 & 8.972 & 12.163 & 0.777 & 7.298 & 10.123 & 0.851 \\
\hline
\end{tabular}

TABLE IX

LR, GPS AND DL-FC MOdEl COMPARISON - TERMINAL 5 - Four EVEnTS

\begin{tabular}{|c|c|c|c|c|c|c|c|c|c|c|c|c|}
\hline \multirow[b]{2}{*}{ Input Data } & \multicolumn{3}{|c|}{ Linear Regression } & \multicolumn{3}{|c|}{ Gaussian Processes } & \multicolumn{3}{|c|}{ Deep GPs } & \multicolumn{3}{|c|}{ DL-FC } \\
\hline & MAE & RMSE & $R^{2}$ & MAE & RMSE & $R^{2}$ & MAE & RMSE & $R^{2}$ & MAE & RMSE & $R^{2}$ \\
\hline Pick-up Lags & 11.700 & 17.804 & 0.586 & 9.164 & 15.932 & 0.698 & 11.211 & 18.694 & 0.544 & 9.406 & 15.629 & 0.681 \\
\hline Pick-up \& Drop-off Lags & 8.332 & 13.462 & 0.764 & 8.701 & 13.265 & 0.770 & 11.279 & 18.739 & 0.542 & 8.133 & 12.160 & 0.774 \\
\hline Pick-up \& Drop-off Lags \& & 8.415 & 13.597 & 0.757 & 8.788 & 13.398 & 0.763 & 11.392 & 18.926 & 0.539 & 8.296 & 12.403 & 0.759 \\
\hline $\begin{array}{l}\text { Pick-up \& Drop-off Lags \& } \\
\text { Weather \& Event info }\end{array}$ & 8.499 & 13.407 & 0.766 & 8.698 & 13.259 & 0.771 & 11.203 & 18.706 & 0.544 & 7.071 & 10.971 & 0.822 \\
\hline $\begin{array}{c}\text { Pick-up \& Drop-off Lags \& } \\
\text { Weather \& Event info \& Topics }\end{array}$ & 8.504 & 13.277 & 0.770 & 8.473 & 12.413 & 0.799 & 11.233 & 18.717 & 0.543 & 6.744 & 10.563 & 0.835 \\
\hline
\end{tabular}

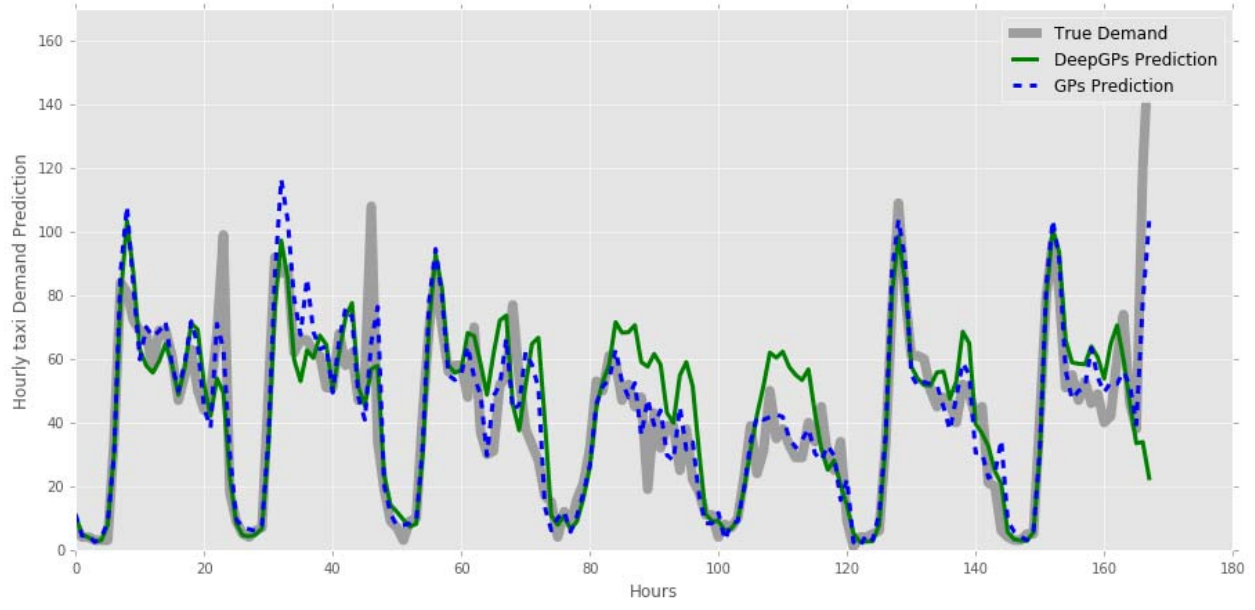

Fig. 5. Demand Prediction using GPs and DeepGPs.

more information about events and their characteristics helps them identify the different patterns between days with events and days with no events and improve their performance by $10.21 \%$ compared to the baseline model with only pickup lags.

In the second case, where we have a week with four events, it appears that Deep GPs are not able to identify correctly the demand peaks. In Figure 5 we can see that Deep GPs predict days with average demand even though an event is organized, and in two cases there is a forecast for high demand during the afternoon hours with no event scheduled. On the other hand, LR and GPs performance is better as shown in Table IX. The introduction of event and topic parameters shows once again demand peaks' effective recognition enhancement.

\section{B. Demand Forecasting Using Fully-Connected Layers}

The data fusion architecture that makes use of fullyconnected (FC) layers for modeling the time-series data is depicted in Figure 6. All the time-series information is provided as a flat input vector to the network in the form of lagged information, following the implemented methodology steps presented in the previous methods. The network is fed with the values for the observations at times $\{t, t-1, \ldots, t-L\}$ in a vector of size $L+1$, where $L+1$ corresponds to the number of lags. This vector is fed into a FC layer with 100-200 hidden units and hyperbolic tangent (tanh) activations, which also can receive additional inputs with other relevant information, such as the event details described in previous subsections. The output of this FC layer is then passed to a second FC layer with 50 units and tanh activations. We apply BatchNormalization [50] before every FC layer, Dropout between FC layers and we use regularization whenever necessary.

The idea is that the output of the last FC layer corresponds to a latent vector representation that encodes all the necessary information form the time-series and other relevant inputs. From this latent vector representation we will finally produce 


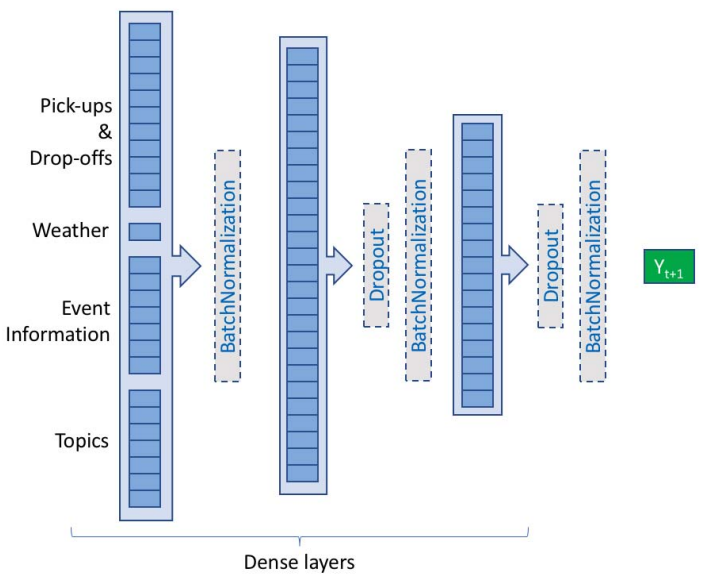

Fig. 6. Proposed neural network architecture with FC layers.

a prediction for $t+1$ using a dense layer. The final prediction is obtained by adding back the removed recurrent trend (based on the historical average) to the output of the neural network.

We kept the same training set of instances as in Deep GPs. For the validation of the proposed architecture, we isolate a separate set of instances. Through this procedure, we keep track of the best performing model during training and we adjust accordingly the majority of the design and hyperparameter choices that the proposed deep learning architectures reflect. The presented architecture was implemented in Keras [51].

The comparison of the proposed methodologies - architectures is concluded with the results of our DL-FC model in Tables VIII and IX. Interestingly, the results show an apparent superiority of the DL-FC model's predictions for the week with four events. With an $R^{2}$ of 0.835 , we conclude that the FC layers can provide us with fairly satisfactory forecasts of days with high demand peaks as well as forecasts of days with demand close the typical daily patterns. Finally, we can once again verify that including information about the events leads to a MAE reduction of $13.1 \%$ and $10.8 \%$ in RMSE. Including the topics in the DL-FC model allows it to further reduce MAE by another $4.6 \%$ and to increase the average $R^{2}$ form 0.822 to 0.835 .

\section{CONCLUSION}

We demonstrated that using online information, we can improve the quality of taxi demand prediction even in scenarios where the transport system is under stress. We combined information extracted from the web with time-series data to build a predictive model of taxi demand around special event venue areas. This is typically a challenging case for transport planning since special events originate high variance in demand. Taxi demand is correlated with many parameters of underlying information and currently, most taxi centers rely on formal processes and manual work for a fleet organization and taxi distribution. Even the more advanced new services, like Uber or Lyft, still face great challenges in terms of demand prediction (shown by price surges and variations thereof). Our results show that by making use of event information from the Web, the proposed models are able to improve the quality of their predictions quite dramatically, thus significantly outperforming other popular time-series forecasting methods from the state of the art that do not account for event information. The proposed approaches make use of the topic modeling advantages that allow the introduction of useful details concerning events' description in our model and from our empirical results the need for accounting events' effect when modeling mobility demand is highlighted.

The initial structure of the model was presented and evaluated using machine learning techniques. Then, each method's performance was compared with a deep learning architecture that is based on fully-connected dense layers, as well as with Deep GPs architecture. In most cases, Gaussian Processes managed to better adapt to the studied venues' demand patterns. Every piece of information, except weather data, contributed positively to the results, thus emphasizing their importance for the formulation of a hourly demand prediction model around a venue.

Noteworthy was also the accuracy of the deep learning architecture. Their predictions' quality improvement is remarkable by making use of event information. At time periods with various events, the presented methodology manages to overcome the alternative models.

In future work, we aim at exploring the impact of spatiotemporal interactions on taxi demand prediction. The development of a city-wide spatio-temporal model that accounts for information about all the events that take place across the city could be a generalization potential of this methodology.

\section{REFERENCES}

[1] Wikipedia, (2017). Uber (Company). Accessed: Nov. 21, 2017. [Online]. Available: https://en.wikipedia.org/wiki/Uber_(company)/

[2] (2017). Grab (Application). Accessed: Nov. 21, 2017. [Online]. Available: https://en.wikipedia.org/wiki/Grab_(application)/

[3] (2017). Beat. [Online]. Accessed: Nov. 21, 2017. [Online]. Available: https://thebeat.co/en/

[4] J. W. Chan, V. L. Chang, W. K. Lau, L. K. Law, and C. J. Lei, "Taxi app market analysis in Hong Kong," J. Econ., Bus. Manage., vol. 4, no. 3, pp. 239-242, 2016.

[5] J. Schmidhuber, "Deep learning in neural networks: An overview," Neural Netw., vol. 61, pp. 85-117, Jan. 2015.

[6] J. Yuan, Y. Zheng, L. Zhang, X. Xie, and G. Sun, "Where to find my next passenger," in Proc. 13th Int. Conf. Ubiquitous Comput., 2011, pp. 109-118.

[7] J. Xu, R. Rahmatizadeh, L. Bölöni, and D. Turgut, "Real-time prediction of taxi demand using recurrent neural networks," IEEE Trans. Intell. Transp. Syst., vol. 19, no. 8, pp. 2572-2581, Aug. 2017.

[8] N. Davis, G. Raina, and K. Jagannathan, "A multi-level clustering approach for forecasting taxi travel demand," in Proc. IEEE ITSC, Nov. 2016, pp. 223-228.

[9] L. Moreira-Matias, J. Gama, M. Ferreira, J. Mendes-Moreira, and L. Damas, "Predicting taxi-passenger demand using streaming data," IEEE Trans. Intell. Transp. Syst., vol. 14, no. 3, pp. 1393-1402, Sep. 2013.

[10] Y. Tong et al., "The simpler the better: a unified approach to predicting original taxi demands based on large-scale online platforms," in Proc. 23rd ACM SIGKDD Int. Conf. Knowl. Discovery Data Mining, 2017, pp. $1653-1662$.

[11] K. Zhang, Z. Feng, S. Chen, K. Huang, and G. Wang, "A framework for passengers demand prediction and recommendation," in Proc. IEEE Int. Conf. Services Comput. (SCC), Jun./Jul. 2016, pp. 340-347.

[12] F. Miao et al., "Taxi dispatch with real-time sensing data in metropolitan areas: A receding horizon control approach," IEEE Trans. Autom. Sci. Eng., vol. 13, no. 2, pp. 463-478, Apr. 2016.

[13] I. Markou, F. Rodrigues, and F. C. Pereira, "Use of taxi-trip data in analysis of demand patterns for detection and explanation of anomalies," Transp. Res. Rec., J. Transp. Res. Board, vol. 2643, pp. 129-138, Sep. 2017. 
[14] E. F. Morgul and K. Ozbay, "Revisiting labor supply of New York City taxi drivers: Empirical evidence from large-scale taxi data," in Proc. TRB 94th Annu. Meeting, no. 15-3331, 2015.

[15] C. Yang and E. J. Gonzales, "Modeling taxi demand and supply in New York City using large-scale taxi gps data," in Seeing Cities Through Big Data. Cham, Switzerland: Springer, 2017, pp. 405-425.

[16] K. Zhao, D. Khryashchev, J. Freire, C. Silva, and H. Vo, "Predicting taxi demand at high spatial resolution: Approaching the limit of predictability," in Proc. IEEE Int. Conf. Big Data, Dec. 2016, pp. 833-842.

[17] M. A. Yazici, C. Kamga, and A. Singhal, 'A big data driven model for taxi drivers' airport pick-up decisions in New York City," in Proc. IEEE Int. Conf. Big Data, Oct. 2013, pp. 37-44.

[18] C. Kamga and M. A. Yazıcı, "Temporal and weather related variation patterns of urban travel time: Considerations and caveats for value of travel time, value of variability, and mode choice studies," Transp. Res. C, Emerg. Technol., vol. 45, pp. 4-16, Aug. 2014.

[19] Y. LeCun, Y. Bengio, and G. Hinton, "Deep learning," Nature, vol. 521, no. 7553 , p. 436, 2015.

[20] Y. Lv, Y. Duan, W. Kang, Z. Li, and F.-Y. Wang, "Traffic flow prediction with big data: A deep learning approach," IEEE Trans. Intell. Transp. Syst., vol. 16, no. 2, pp. 865-873, Apr. 2015.

[21] X. Ma, Z. Tao, Y. Wang, H. Yu, and Y. Wang, "Long short-term memory neural network for traffic speed prediction using remote microwave sensor data," Transp. Res. C, Emerg. Technol., vol. 54, pp. 187-197, May 2015.

[22] J. Zhang, Y. Zheng, and D. Qi, "Deep Spatio-Temporal residual networks for citywide crowd flows prediction," in Proc. AAAI, 2017, pp. $1655-1661$.

[23] A. C. Damianou and N. D. Lawrence, "Deep Gaussian processes," Artif. Intell. Statist., 2013, pp. 207-215.

[24] H. Salimbeni and M. Deisenroth, "Doubly stochastic variational inference for deep Gaussian processes," in Proc. Adv. Neural Inf. Process. Syst., 2017, pp. 4588-4599.

[25] F. C. Pereira, F. Rodrigues, and M. Ben-Akiva, "Internet as a sensor: A case study with special events," in Proc. 91st Annu. Meeting Transp. Res. Board, Washington, DC, USA, Jan. 2012.

[26] F. C. Pereira, F. Rodrigues, E. Polisciuc, and M. Ben-Akiva, "Why so many people? Explaining nonhabitual transport overcrowding with Internet data," IEEE Trans. Intell. Transp. Syst., vol. 16, no. 3, pp. 1370-1379, Jun. 2015.

[27] D. Thom, H. Bosch, S. Koch, M. Wörner, and T. Ertl, "Spatiotemporal anomaly detection through visual analysis of geolocated Twitter messages," in Proc. IEEE Pacific Vis. Symp. (PacificVis), Feb./Mar. 2012, pp. $41-48$.

[28] T. Sakaki, M. Okazaki, and Y. Matsuo, "Earthquake shakes Twitter users: Real-time event detection by social sensors," in Proc. 19th Int. Conf. World Wide Web, 2010, pp. 851-860.

[29] V. Frias-Martinez, V. Soto, H. Hohwald, and E. Frias-Martinez, "Characterizing urban landscapes using geolocated tweets," in Proc. Int. Conf. Privacy, Security, Risk Trust Int. Conf. Social Comput. (SocialCom), 2012, pp. 239-248.

[30] Z. Cheng, J. Caverlee, K. Lee, and D. Z. Sui, "Exploring millions of footprints in location sharing services," in Proc. ICWSM, 2011, pp. 81-88.

[31] B. Hawelka, I. Sitko, E. Beinat, S. Sobolevsky, P. Kazakopoulos, and C. Ratti, "Geo-located Twitter as proxy for global mobility patterns," Cartogr. Geogr. Inf. Sci., vol. 41, no. 3, pp. 260-271, 2014.

[32] D. M. Blei, A. Y. Ng, and M. I. Jordan, "Latent Dirichlet allocation," J. Mach. Learn. Res., vol. 3, pp. 993-1022, Mar. 2003.

[33] F. C. Pereira, F. Rodrigues, and M. Ben-Akiva, "Using data from the Web to predict public transport arrivals under special events scenarios," J. Intell. Transp. Syst., vol. 19, no. 3, pp. 273-288, 2015.

[34] K. Kireyev, L. Palen, and K. M. Anderson, "Applications of topics models to analysis of disaster-related Twitter data," in Proc. NIPS Workshop Appl. Topic Models, Text Beyond, vol. 1, Whistler, Canada, 2009, pp. 1-4.

[35] D. Ramage, S. Dumais, and D. Liebling, "Characterizing microblogs with topic models," Proc. ICWSM, vol. 10, 2010, pp. 1-8.

[36] H. Ma, B. Wang, and N. Li, "A novel online event analysis framework for micro-blog based on incremental topic modeling," in Proc. SNPD, 2012, pp. 73-76.

[37] (2017). TLC Trip Record Data. Accessed: Nov. 21, 2017. [Online]. Available: http://www.nyc.gov/html/tlc/html/about/trip_record_data.shtml

[38] (2017). Best Concert Venues in NYC. Accessed: Aug. 14, 2017. [Online]. Available: https://www.nyc.com/nyc-guides/best_concert venues in nyc.308/
[39] F. Pedregosa et al., "Scikit-learn: Machine learning in Python," J. Mach. Learn. Res., vol. 12, pp. 2825-2830, Oct. 2011.

[40] T. Idé and S. Kato, "Travel-time prediction using Gaussian process regression: A trajectory-based approach," in Proc. SIAM Int. Conf. Data Mining, 2009, pp. 1185-1196.

[41] Y. Xie, K. Zhao, Y. Sun, and D. Chen, "Gaussian processes for shortterm traffic volume forecasting," Transp. Res. Rec., J. Transp. Res. Board, vol. 2165, no. 1, pp. 69-78, 2010.

[42] F. Rodrigues, S. S. Borysov, B. Ribeiro, and F. C. Pereira, "A Bayesian additive model for understanding public transport usage in special events," IEEE Trans. Pattern Anal. Mach. Intell., vol. 39, no. 11, pp. 2113-2126, Nov. 2017.

[43] C. E. Rasmussen, "Gaussian processes in machine learning," in Advanced Lectures on Machine Learning. Berlin, Germany: Springer, 2004, pp. 63-71.

[44] M. Seeger, "Gaussian processes for machine learning," Int. J. Neural Syst., vol. 14, no. 2, pp. 69-106, 2004.

[45] F. Rodrigues, I. Markou, and F. C. Pereira, "Combining time-series and textual data for taxi demand prediction in event areas: A deep learning approach," Inf. Fusion, vol. 49, pp. 120-129, Sep. 2018.

[46] F. A. Gers, D. Eck, and J. Schmidhuber, "Applying LSTM to time series predictable through time-window approaches," in Neural Nets WIRN Vietri-01. London, U.K.: Springer, 2002, pp. 193-200.

[47] Q. Cheng, Y. Liu, W. Wei, and Z. Liu, "Analysis and forecasting of the day-to-day travel demand variations for large-scale transportation networks: A deep learning approach," Transportation Analytics Contest, Tech. Rep., 2016. doi: 10.13140/RG.2.2.12753.53604

[48] D. Duvenaud, O. Rippel, R. P. Adams, and Z. Ghahramani, "Avoiding pathologies in very deep networks," in Proc. 17th Int. Conf. Artif. Intell. Statist., (AISTATS), Reykjavik, Iceland, Apr. 2014, pp. 202-210.

[49] J. Snoek, H. Larochelle, and R. P. Adams, "Practical Bayesian optimization of machine learning algorithms," in Proc. Adv. Neural Inf. Process. Syst., 2012, pp. 2951-2959.

[50] S. Ioffe and C. Szegedy. (2015). "Batch normalization: Accelerating deep network training by reducing internal covariate shift." [Online]. Available: https://arxiv.org/abs/1502.03167

[51] F. Chollet, "Keras," GitHub Repository, Tech. Rep., 2015. [Online]. Available: https://github.com/fchollet/keras

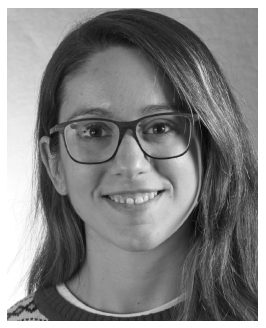

Ioulia Markou is currently pursuing the Ph.D degree in management engineering with the Technical University of Denmark. She is currently focused on understanding urban mobility with a particular emphasis on the influence of special events in the transport system. Her research interests include machine learning, intelligent transportation systems, and modeling and simulation of transportation systems and urban mobility.

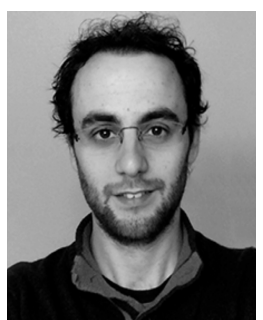

Filipe Rodrigues received the Ph.D. degree in information science and technology from the University of Coimbra, Portugal. He is currently a PostDoctoral Fellow with the Technical University of Denmark, where he is working on machine learning models for understanding urban mobility and the behavior of crowds, with an emphasis on the effect of special events in mobility and transportation systems. His research interests include machine learning, probabilistic graphical models, natural language processing, intelligent transportation systems, and urban mobility.

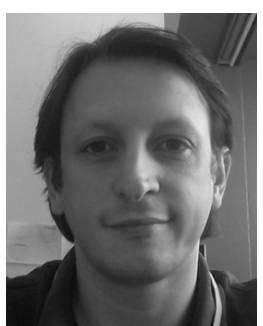

Francisco C. Pereira received the master's and $\mathrm{Ph} . \mathrm{D}$. degrees in computer science from the University of Coimbra, in 2000 and 2005, respectively. $\mathrm{He}$ is currently a Full Professor with the Technical University of Denmark, where he leads the Smart Mobility Research Group. His main research focus is on applying machine learning and pattern recognition in the context of transportation systems with the purpose of understanding and predicting mobility behavior, and modeling and optimizing the transportation system as a whole. 